\title{
Learning-set formation in the bottlenose dolphin'
}

LOUIS M. HERMAN, FRANK A. BEACH, III, ROSS L. PEPPER, and RICHARD B. STALLING, University of Hawaii, Honolulu, Hawaii 96822

Formation of a discrimination-learning set was investigated in the Pacific bottlenose dolphin over 80 two-choice problems. Although average Trial-2 performance compared well with some primate species, performance level on later trials and on successive blocks of problems did not progressively improve. Spatial separation of stimulus cue and response locus was discussed as a possible contributor to the observed limited performance level.

Although there has been much speculative interest in dolphin "intelligence," stemming in part from analysis of its neuroanatomical features, and in part from observation of its natural behaviors, there has been little empirical work which bears directly on its complex-learning ability. The many studies of the auditory sensory capabilities of the dolphin (e.g., Norris, 1966), though indicative of a high level of discriminative capacity for this sensory system, do not provide necessary information for assessing the learning ability of the dolphin relative to other higher mammals. The present study was an attempt to assess this ability through investigation of the dolphin's performance in attainment of a discrimination-learning set.

\section{METHOD}

The subject of study was Wela, a female Pacific bottlenose dolphin (Tursiops gillii) of approximately 10-12 years of age and $350 \mathrm{lbs}$. She had been in captivity at Sea Life Park in Hawaii for approximately three years, and had served primarily in demonstrations, retrieving objects and carrying them to a diver. She was in good health, worked well for fish reward during the prolonged testing sessions of this study, and rarely showed behavior that could be classified "emotional." Testing was in a large, oval sea-water tank in which the animal lived.

The contour-stimuli were various unique geometrical or abstract shapes, cut from 1-ft square plywood and painted white with a red 1 -in. border to increase contour contrast through differential brightness. Contours were used to permit discrimination by visual and/or auditory means. Equipment was not available for monitoring of sonic outputs of the dolphin, however, and the degree of use of this system in achieving discriminations could not be ascertained.

The two contours for each problem were presented to the animal approximately $6 \mathrm{ft}$ apart and $18 \mathrm{in}$. below the water surface, each attached to a $4 \frac{1}{2}-\mathrm{ft}$ movable lever arm which projected into the water from a center shaft. Position of the arms could be reversed and the arms readily removed from the water between trials. For the animal, the only visible (unscreened) portions of the apparatus were the two contours when immersed in the water.

Discrimination training was introduced using an "errorless" training technique, but all subsequent test problems were presented using standard discrimination procedures. For half the problems, the stimulus Wela responded to on Trial 1 was designated St, and, for the remaining problems, it was designated $\mathrm{S}-$. A total of 12 trials per problem was presented over the first 40 problems, but the remaining 40 were shortened to either six or eight trials to permit additional problem testing within the limited time available.

Following initial discrimination training, Wela was trained to approach the apparatus only during presentation of a light, located between the paired contours, and a 600-cps tone of $6-\mathrm{sec}$ duration. The discrimination response required of Wela was to press one of the contour targets with her rostrum, thereby displacing the lever arm and tripping a switch. Closing of the correct switch (i.e., pressing $\mathrm{S}+$ ) produced immediate offset of the light and onset of a 4800-cps conditioned-reinforcement tone. Closing the incorrect switch produced only offset of the light and terminated the trial.

Following closing of a switch the contours were removed from view and Wela returned to the opposite end of the tank either to receive a fish reward or to station herself. Intertrial interval was approximately $30 \mathrm{sec}$, and interproblem interval was approximately $7 \mathrm{~min}$. Testing was spaced over eight days within a three-week period.

\section{RESULTS}

Table 1 shows the percent-correct responses achieved by Wela on each trial over 10 successive blocks of eight problems. It can be seen that a general lack of improvement over successive trials within blocks characterized Wela's performance. Though average performance during early trials was good-57.2\% on Trial 2, and up to $60 \%$ on Trials 3 and 4-performance on later trials fluctuated about these values. A lack of progressive improvement across problems, shown in Table 1 by the mean per cent correct for blocks, also characterized Wela's performance. An additional feature of Wela's behavior noted was the development of a strong position habit. Position responses were never below $67 \%$ and tended to increase as problems progressed.

\section{DISCUSSION}

In evaluating both early- and late-trial performance, a study by Miles \& Meyer (1956) provides a ready means for comparison of dolphin performance with primate behavior. In the cited study, discrimination-learning set performance of the rhesus macaque and of the more primitive marmoset were compared over 98 six-trial problems. Trial-2 performance for the rhesus was $60 \%$ correct, and for the marmoset only $50 \%$ correct. In the present study, Trial-2 performance level of the dolphin over 80 problems was $57 \%$ correct. On Trial 6, however, the rhesus averaged $80 \%$ correct, the marmoset $58 \%$, and the dolphin approximately 59\%. Though such comparisons should be considered only preliminary, it would seem that the early performance level of the dolphin should have promised considerably better later-trial performance than was actually observed. Since only one prior study (Kellogg \& Rice, 1964) of complex learning-though not learning set-has been reported with this animal, there is little empirical

Table 1

Per Cent Correct Per Trial for Successive Blocks of Eight Problems Block

\begin{tabular}{cccccccccccc}
\cline { 2 - 3 } & 1 & 2 & 3 & 4 & 5 & 6 & 7 & 8 & 9 & 10 & Mean \\
\hline 2 & 62.5 & 50.0 & 87.5 & 37.5 & 75.0 & 25.0 & 62.5 & 87.5 & 50.0 & 37.5 & 57.2 \\
3 & 62.5 & 37.5 & 87.5 & 50.0 & 62.5 & 62.5 & 75.0 & 50.0 & 62.5 & 50.0 & 60.0 \\
4 & 75.0 & 37.5 & 75.0 & 62.5 & 75.0 & 62.5 & 37.5 & 62.5 & 50.0 & 62.5 & 60.0 \\
5 & 50.0 & 75.0 & 62.5 & 50.0 & 25.0 & 25.0 & 37.5 & 50.0 & 25.0 & 37.5 & 43.8 \\
6 & 62.5 & 50.0 & 87.5 & 50.0 & 62.5 & 75.0 & 75.0 & 37.5 & 50.0 & 37.5 & 58.8 \\
7 & 62.5 & 50.0 & 50.0 & 62.5 & 62.5 & & & & 37.5 & 62.5 & 55.4 \\
8 & 50.0 & 37.5 & 87.5 & 37.5 & 50.0 & & & & 50.0 & 50.0 & 51.8 \\
9 & 50.0 & 75.0 & 50.0 & 50.0 & 75.0 & & & & & & 60.0 \\
10 & 37.5 & 37.5 & 75.0 & 75.0 & 37.5 & & & & & & 52.5 \\
11 & 50.0 & 62.5 & 62.5 & 87.5 & 62.5 & & & & & & 65.0 \\
12 & 37.5 & 37.5 & 37.5 & 25.0 & 50.0 & & & & & & 37.5 \\
\hline
\end{tabular}

$\begin{array}{llllllllll}\text { Mean } 54.5 & 50.0 & 69.3 & 53.4 & 59.9 & 50.0 & 57.5 & 57.5 & 46.4 & 48.2\end{array}$ 
information on optimal test features. Some improvement in test performance may occur as these features are uncovered and implemented in future research.

One feature which may be identified now, however, concerns the distance between the relevant stimulus cue, i.e., the borders of the stimulus figures, and the locus at which the dolphin made its pressing response. Wela tended to press the contour-stimuli at or near their centers, which were uniform in appearance across stimuli, and offered no relevant cue. Thus, there was spatial discontiguity between locus of the relevant cue at the borders and locus of the pressing response. Meyer et al (1965) report that displacement of the relevant cue by as little as .5 in. from the locus of response results in considerable drop in discrimination-learning set performance in monkeys. Stollnitz (1965) has provided a theoretical basis for this decrement in terms of a failure to reinforce the observing response, i.e., the response of attending to the relevant cue, in problems characterized by spatial discontiguity and by relatively few trials. He notes that monkeys tend to look where they put their fingers, and animals lacking fingers look at what their noses touch. If the observing response is not strengthened, then within-problem learning is retarded and transfer of learning across problems is precluded. In this case, some irrelevant stimulus dimension, such as spatial position, may come to control behavior.

Examination of the degree of spatial discontiguity for the contour pairs used in the first 40 12-trial problems of the present study provided a partial check on the assumption that this variable was an important determinant of the dolphin's performance level. In 14 of the 40 problems, for Trials 2 to 12 , discrimination performance-though not necessarily learning-could be classified as "good." These were problems in which either the $\mathbf{S}+$ stimulus was chosen in at least 9 of the 11 trials $(p<.06)$, or the $S$ - stimulus was so chosen. The remaining 26 problems were classified as "poorer" discrimination performance. Measurements taken of the minimum distance from the center of each contour stimulus to its border revealed that the average minimum distance was smaller by .6 in. for the "good" problems. As has been noted, this is a sufficient spatial discontiguity to have detrimental effects on primate performance.

It appears, therefore, that there are both empirical and theoretical grounds for concluding that spatial discontiguity between stimulus-cue locus and response locus could have been of major importance in restricting the dolphin's performance level in this study. Research is currently in progress to investigate this variable and to assess further the dolphin's ability to form learning sets.

\section{REFERENCES}

KELLOGG, W., \& RICE, C. Visual problem-solving in a Bottlenose Dolphin. Science, 1964, 143, 1053-1055.

MEYER, D. R., TREICHLER, F. R., \& MEYER, P. M. Discrete-trial training and stimulus variables. In A. M. Schrier, H. F. Harlow, and F. Stollnitz (Eds.), Behavior of nonhuman primates. Vol 1. New York: Academic Press, 1965. Chap. 1.

MILES, R. C., \& MEYER, D. R. Learning sets in marmosets. Journal of Comparative \& Physiological Psychology, 1956, 49, $219-222$.

NORRIS, K. S. (Ed.) Whales, dolphins, and porpoises. Berkeley: University of California Press, 1966.

STOLLNITZ, F. Spatial variables, observing responses, and discrimination learning sets. Psychological Review, 1965, 72, 247-261. NOTE

1. This research was supported in part by a grant from Oceanic Institute, Oahu. Appreciation is extended to Sea Life Park, Oahu, for generous use of their facilities.

\section{(Continued from page 97)}

\section{DISCUSSION}

Thompson \& Rich's (1962) basic finding, that of differential performance on black vs dark-grey (brightness) and pattern discriminations after npt lesions, was confirmed. The differences do not originate through measurement artifact.

The postoperative reaction to shock, as observed in this study, suggests that at least some portion of the postoperative deficit may be attributed to the level of shock used. Further evidence for the importance of motivational factors was provided by Breen \& Thompson (1966), who have shown that postoperative deficits of npt-lesioned animals are minimal if they are tested under thirst motivation and Thompson et al (1967) who, using mild shock, reported results contradicting both this and Thompson \& Rich's (1962) study. Also of relevance might be Myers' (1964) report that lesions in the posterior thalamic area in the cat produce marked visual unresponsiveness.

In addition to motivational factors, sensory factors, possibly interacting with the former to produce postoperative deficits, must also be involved in npt-lesion effects. This is indicated by the increasing difficulties Ss had in solving brightness discriminations of increasing difficulty (Tasks $A$ to $E$ ) postoperatively. It might be argued that the postoperative errors simply reflect the difficulty Ss had in learning the discrimination originally; this is negated by the performance of Groups $\mathrm{C}$ and $\mathrm{F}$ and by the fact that all control Ss, regardless of the discrimination, showed perfect retention over the postoperative recovery interval. Thus, the differential performance on Tasks $\mathrm{C}$ and $\mathrm{F}$ might be interpreted as reflecting a sensory disturbance which somehow affects $\mathrm{C}$ more adversely than F. Support for such a possibility is provided by the finding that naive npt-lesioned Ss trained to learn either $\mathrm{C}$ or $\mathrm{F}$ also show the differences described previously for the training-operation-retraining situation (Peters, M., unpublished, $\mathrm{N}_{1}=5, \mathrm{~N}_{2}=6, \mathrm{p}=.015$, by Mann-Whitney $\mathrm{U}$ test; stripes performance superior).

The above interpretation of npt lesion deficits in terms of motivational and sensory factors stands in contrast to Thompson's (1965) stress on memory factors. It is felt, however, that before elusive factors such as memory are considered, the role of the more tangible motivational and sensory factors in $\mathrm{npt}$ lesion deficits should be thoroughly explored. Currently, attempts are made to experimentally define those stimulus dimensions which differentiate between discriminations $\mathrm{C}$ and $\mathrm{F}$. If such stimulus dimensions can be defined it might be possible to deduce the sensory impairments following npt lesions.

\section{REFERENCES}

BREEN, T., \& THOMPSON, R. Cortical and subcortical structures mediating a visual conditioned response motivated by thirst. Journal of Comparative \& Phy siological Psychology, 1966, 61, 146-150.

De GROOT, J. The rat forebrain in stereotax ic coordinates. Amsterdam: N.V. Nord-Hollandsche Uitgevers, Maatschappij, 1959.

MYERS, R. E. Visual deficits after lesions of the brain stem tegmentum in cats. Archives of Neurology, 1964, 11, 73-90.

THOMPSON, R. Centrencephalic theory and inter-hemispheric transfer of visual habits. Psychological Review, 1965, 72, 385-398.

THOMPSON, R., \& RICH, I. Differential effects of posterior thalamic lesions on retention of various visual habits. Journal of Comparative $\&$ Physiological Psychology, 1963, 56, 60-65.

THOMPSON, R., LUKASZEWSKA, I., SCHWEIGERDT, A., \& McNEW, $J$. Retention of visual and kinesthetic discriminations in rats following pretecto-diencephalic and ventral mesencephalic damage. Joumal of Comparative \& Physiological Psychology, 1967, 63, 458-468. NOTES

1. This study was supported by NRC Grant APA-135.

2. Now at University of Western Ontario, London, Ontario, Canada. 